\title{
GROUPS WHOSE DEGREE GRAPH HAS THREE INDEPENDENT VERTICES
}

\author{
SILVIO DOLFI, KHATOON KHEDRI, AND EMANUELE PACIFICI
}

\begin{abstract}
Let $G$ be a finite group, and let $\operatorname{cd}(G)$ denote the set of degrees of the irreducible complex characters of $G$. This paper is a contribution to the study of the degree graph of $G$, that is, the prime graph built on the set $\operatorname{cd}(G)$. Namely, we characterize finite groups whose degree graph has three independent vertices (i.e., three vertices that are pairwise non-adjacent). Our result turns out to be a generalization of several previously-known theorems concerning the structure of the degree graph.
\end{abstract}

\section{INTRODUCTION}

Let $G$ be a finite group. A well-established research field in Character Theory is the study of the set $\operatorname{cd}(G)$, whose elements are the degrees of the irreducible complex characters of $G$. In fact, many results in the literature show that even this relatively small set of positive integers encodes nontrivial information about the structure of $G$; in particular, there is a significant interplay between the group structure and the "arithmetical structure" of $\operatorname{cd}(G)$ (i.e., the way in which the numbers in this set decompose into prime factors).

An important tool that has been devised in order to analyze this aspect of $\operatorname{cd}(G)$, is the degree graph $\Delta(G)$ of $G$; this is the simple undirected graph whose vertex set $\mathrm{V}(G)$ consists of all the prime numbers that divide some element in $\operatorname{cd}(G)$, while a subset $\{p, q\}$ of $\mathrm{V}(G)$ belongs to the edge set $\mathrm{E}(G)$ if and only if $p q$ divides an element in $\operatorname{cd}(G)$.

In the case when $G$ is a finite solvable group, a fundamental theorem by P.P. Pálfy ([19]) establishes that the size of a set of independent vertices in $\Delta(G)$ is at most two; in other words, if $\pi$ is a subset of $\mathrm{V}(G)$ such that $|\pi| \geq 3$, then there exist $p, q \in \pi$ such that $\{p, q\}$ belongs to $\mathrm{E}(G)$. Another equivalent formulation is the following: if $G$ is solvable, then the complement graph of $\Delta(G)$ contains no triangles. As immediate consequences, the number of connected components of $\Delta(G)$ is at most 2; if $\Delta(G)$ is disconnected, then each connected component induces a complete subgraph of $\Delta(G)$, whereas, in the connected case, the diameter of $\Delta(G)$ is at most three. (We note that Palfy's result has been recently extended by Z. Akhlaghi, C. Casolo and the authors in [1]: for a finite solvable group $G$, the complement graph $\Delta(G)$ does not contain any cycle of odd length, and it is therefore a bipartite graph.)

As for non-solvable groups, the situation is different; while the diameter of $\Delta(G)$ (in the connected case) turns out to be at most three for any finite group $G$ ([14]

2000 Mathematics Subject Classification. 20C15.

The first and third author were partially supported by the Italian PRIN 2015TW9LSR_006 "Group Theory and Applications". 
and $[15])$, this graph may have sets of independent vertices whose size is larger than two. For instance, the degree graph of the alternating group Alt(5) is an empty triangle and, in general, $\Delta\left(\mathrm{PSL}_{2}\left(2^{a}\right)\right)$ has three connected components for every $a \geq 2$ (see Proposition 2.6). Nevertheless, a universal bound for the size of a set of independent vertices in $\Delta(G)$ was established by A. Moretó and P.H. Tiep in [18], and this bound turns out to be in fact three. Therefore, three is also the maximum number of connected components of $\Delta(G)$.

The aim of the present paper is to investigate in which respect the structure of a finite group $G$ is influenced by the existence of three independent vertices in $\Delta(G)$. The relevance of this aspect might be suggested by the fact that the finite groups whose degree graph has three connected components are completely characterized as direct products of the kind $\mathrm{PSL}_{2}\left(2^{a}\right) \times A$, where $a \geq 2$ and $A$ is an abelian finite group (see $[13]$ ). The main result of the paper is the following.

Theorem A. Let $G$ be a finite group, and let $\pi \subseteq \mathrm{V}(G)$ be such that $|\pi|=3$. Then $\pi$ is an independent set of $\Delta(G)$ if and only if $\mathbf{O}^{\pi^{\prime}}(G)=S \times A$, where $A$ is abelian and $S \simeq \mathrm{SL}_{2}\left(p^{a}\right)$ or $S \simeq \operatorname{PSL}_{2}\left(p^{a}\right)$, for a prime $p \in \pi$ and positive integer $a$. Moreover, writing $\pi=\{p, q, s\}$, then $q$ divides $p^{a}+1, s$ divides $p^{a}-1$ and $q, s$ are both odd primes.

Since, in the setting of Theorem A, both $p^{a}+1$ and $p^{a}-1$ have odd prime divisors, we observe that the cardinality $p^{a}$ of the defining field can neither be a Mersenne nor a Fermat prime and that $p^{a} \neq 2,9$.

As more or less immediate consequences of Theorem A, we derive some of the aforementioned (and previously known) results. In particular, we obtain the bound of three for the size of an independent set of vertices, as well as the bound of three for the diameter of the degree graph of any finite group (in the connected case).

Corollary B. Let $G$ be a finite group, and $\pi$ a set of vertices of $\Delta(G)$ with $|\pi|=4$. Then there exist two vertices in $\pi$ that are adjacent in $\Delta(G)$.

Corollary C. Let $G$ be a finite group such that $\Delta(G)$ is connected. Then the diameter of $\Delta(G)$ is at most three.

Finally, we also re-obtain the characterization of finite groups whose degree graph has three connected components.

Corollary D. Let $G$ be a finite group. Then $\Delta(G)$ has three connected components if and only if $G \simeq \operatorname{PSL}_{2}\left(2^{a}\right) \times A$ with $A$ abelian.

We conclude by mentioning that every group considered in the following discussion is assumed to be a finite group.

\section{Proofs}

We start with some results concerning some relevant properties of group actions on finite modules. The following setting arises naturally in problems related to coprimality with a fixed prime $q$ of all degrees of irreducible characters lying over characters of a minimal normal subgroup which are extendible to their inertia subgroup.

Let $H$ and $V$ be finite groups, and assume that $H$ acts by automorphisms on $V$. Given a prime number $q$, we say that the pair $(H, V)$ satisfies $\mathcal{N}_{q}$ if

(a) $q$ divides $\left|H: \mathbf{C}_{H}(V)\right|$ and 
(b) for every non-trivial $v \in V$, there exists a Sylow $q$-subgroup $Q$ of $H$ such that $Q \unlhd \mathbf{C}_{H}(v)$.

We refer to [2] for a thorough analysis of this and related module actions. We recall that, as shown for instance by Lemma 4 of [22], if $(H, V)$ satisfies $\mathcal{N}_{q}$, then $V$ is an elementary abelian $r$-group for a suitable prime $r$ and that $V$ is an irreducible $\mathrm{GF}(r)[H]$-module.

Note also that, if $K$ is a normal subgroup of $H$ such that $q$ divides $\left|K / \mathbf{C}_{K}(V)\right|$, then $(K, V)$ satisfies $\mathcal{N}_{q}$ as well.

Let $r$ be a prime number and $n$ a positive integer; as customary, we denote by $\Gamma\left(r^{n}\right)$ the semilinear group on the field $\operatorname{GF}\left(r^{n}\right)$, and by $\Gamma_{0}\left(r^{n}\right)$ the subgroup of $\Gamma\left(r^{n}\right)$ induced by the field multiplications. Given a group $G$ and a faithful $G$ module $V$ over $\operatorname{GF}(r), r$ a prime, we say that $G$ is a semilinear group on $V$, and write $G \leq \Gamma(V)$, if there exists an injective homomorphism $\phi: G \rightarrow \Gamma\left(r^{n}\right)$, where $r^{n}=|V|$, such that the additive group $\mathrm{GF}\left(r^{n}\right)^{+}$of $\mathrm{GF}\left(r^{n}\right)$ (with the $G$-module structure carried by $\phi$ ) and $V$ are isomorphic $G$-modules. In this setting, given $H \leq G$, we write $H \leq \Gamma_{0}(M)$ if $\phi(H) \leq \Gamma_{0}\left(r^{n}\right)$ for $\phi$ as above.

Lemma 2.1. Let $K$ be a subgroup of $\Gamma(V),|V|=r^{n}, r$ a prime. Write $K_{0}=$ $K \cap \Gamma_{0}(V)$. Assume that $(K, V)$ satisfies $\mathcal{N}_{q}$. Then

(a) $q$ divides $n,\left(r^{n}-1\right) /\left(r^{n / q}-1\right)$ divides $\left|K_{0}\right|$ and $\left(q, r^{n}-1\right)=1$;

(b) $q$ divides $|K / \mathbf{F}(K)|$.

Proof. (a) follows from Lemma 3.5 in [4]. (b) is immediate, by observing that condition $\mathcal{N}_{q}$ implies that $\mathbf{O}_{q}(K) \leq \mathbf{C}_{K}(V)=1$.

Theorem 2.2. Let $H$ be a group, $r$ a prime, and $V$ a faithful $\mathrm{GF}(r)[H]$-module of order $r^{n}$. Let $K$ be a normal subgroup of $H$ and assume that, for a prime $q$, the pair $(K, V)$ satisfies $\mathcal{N}_{q}$. Then the following conclusions hold.

(a) If $K$ has a normal $q$-complement and $r \neq q$, then $H \leq \Gamma(V)$.

(b) If $K$ is solvable, then either (i) $H \leq \Gamma(V)$, or (ii) $r=q=3,|V|=3^{2}$ and $H \simeq \mathrm{SL}_{2}(3)$ or $H \simeq \mathrm{GL}_{2}(3)$.

Proof. In the setting of (a), we can assume that the Sylow $q$-subgroups of $K$ are abelian; in fact, denoting by $U$ be the normal $q$-complement of $K$ and by $Q$ a Sylow $q$-subgroup of $K$, the subgroup $K_{0}=U \mathbf{Z}(Q)$ is characteristic in $K$ (hence normal in $H)$, and the pair $\left(K_{0}, V\right)$ satisfies $\mathcal{N}_{q}$. Therefore, we can use $K_{0}$ in the role of $K$. As there is no loss of generality in this replacement, with a slight abuse, we keep denoting this subgroup by $K$.

Now, for $v \in V$, let $Q \in \operatorname{Syl}_{q}(K)$ be such that $Q \unlhd \mathbf{C}_{K}(v)$. Since $\mathbf{C}_{K}(v)$ has a normal $q$-complement and $Q \unlhd \mathbf{C}_{K}(v)$ is abelian, then $Q$ is central in $\mathbf{C}_{K}(v)$; as $r \neq q$, the Main Theorem of [2] implies $K \leq \Gamma(V)$.

On the other hand, if $K$ is solvable (as in the assumption of (b)) then, by [2, Corollary 10], either conclusion (ii) of (b) holds, or $K \leq \Gamma(V)$ as well. Thus, we will finish the proof starting from the assumption $K \leq \Gamma(V)$ and showing that $H \leq \Gamma(V)$.

Let $K_{0}=K \cap \Gamma_{0}(V)$. By Lemma 2.1, we get that $q$ does not divide $\left|\Gamma_{0}(V)\right|=$ $r^{n}-1$; moreover, $q$ divides $n$ and $\left(r^{n}-1\right) /\left(r^{n / q}-1\right)$ divides $\left|K_{0}\right|$.

Assume first that the pair $(r, n)$ does not have a primitive prime divisor: then, by Zsigmondy's Theorem, either $r$ is a Mersenne prime, i.e. $r=2^{k}-1$ for a suitable $k \geq 2$ and $n=2$, or $(r, n)=(2,6)$. 
In the former case, the arithmetical conditions recalled above imply $q=2$ and $r+1|| K_{0} \mid$; but then $2^{k}$ divides $\left|K_{0}\right|$, a contradiction as $q=2$ does not divide $\left|\Gamma_{0}(V)\right|$. On the other hand, assume $(r, n)=(2,6)$; in this case we get $q=2$ and 9||$K_{0} \mid$. In particular, $K$ has a cyclic subgroup $C$ of order 9 , which turns out to be characteristic in $K$ as $C$ is the unique Sylow 3-subgroup of $\mathbf{O}^{3}(K)$ (a subgroup of $\left.\mathbf{O}^{3}\left(\Gamma\left(2^{6}\right)\right) \simeq D_{18} \times C_{7}\right)$. As a consequence, $C$ is normal in $H$ and, since the action of $C$ on $V$ is clearly irreducible, an application of [17, Theorem 2.1] yields $H \leq \Gamma(V)$, as wanted.

In view of the above discussion, we can assume that there exists a primitive prime divisor $t$ for the pair $(r, n)$, and therefore $t$ divides $\left|K_{0}\right|$. If $T_{0}$ is the subgroup of order $t$ of $K_{0}$, clearly $T_{0}$ is a characteristic subgroup of $K$ and hence $T_{0} \unlhd H$. Moreover, since $t$ is a primitive prime divisor of $(r, n)$, the action of $T_{0}$ on $V$ is irreducible. Therefore, as above, an application of Theorem 2.1 in [17] completes the proof.

In what follows, given a $G$-module $V$ and a subgroup $H$ of $G$, we will use the (slightly shorter) notation $V_{H}$ for $\mathbf{C}_{V}(H)$.

Proposition 2.3. Let $(G, V)$ satisfy $\mathcal{N}_{q}$, and let $Q$ be a Sylow q-subgroup of $G$. Let $|V|=r^{a},\left|V_{Q}\right|=r^{b}$ (where $r$ is a prime and $a, b$ positive integers). Then the following hold.

(a) If $q=2$, then $r=2$.

(b) $\left|G: \mathbf{N}_{G}(Q)\right|=\frac{r^{a}-1}{r^{b}-1}$.

(c) $b$ divides $a$ and $1 \leq b<a$; if $q \neq r$, then $b<a / 2$.

(d) If $r \notin\{2, q\}$, then there exists a primitive prime divisor $t$ of $r^{a}-1$ and $t$ divides $|G|$.

Proof. Claims (a) and (b) are (2) and (3), respectively, of [2, Proposition 8]. As regards (c), it follows from (b) that $b$ divides $a$; note that $b=a$ implies $Q \leq \mathbf{C}_{G}(V)$, against the assumption $q|| G / \mathbf{C}_{G}(V) \mid$. If $b=a / 2$, then $\left|G: \mathbf{N}_{G}(Q)\right|=r^{a / 2}+1$ and hence $r=q$.

Consider finally Claim (d). Since $r \neq q$, by (c) we have $a \neq 2$; moreover, as $r \neq 2$, then $r^{a} \neq 2^{6}$. By Zsigmondy's Theorem, there exists a primitive prime divisor $t$ of $r^{a}-1$ and, by (b), $t$ divides $\left|G: \mathbf{N}_{G}(Q)\right|$.

The next lemma is similar to Lemma 14 of [2].

Lemma 2.4. Assume that $(G, V)$ satisfies $\mathcal{N}_{q}$ and let $K$ be a normal subgroup of $G$ with $|K|$ coprime to $q$. Let $X \leq K$ and let $Y=\mathbf{C}_{G}(X)$. If $q$ divides $\left|G: \mathbf{C}_{G}\left(V_{X}\right)\right|$, then $\left(Y, V_{X}\right)$ satisfies $\mathcal{N}_{q}$.

Proof. Since $\mathbf{C}_{G}\left(V_{X}\right) \neq G$, then $V_{X} \neq 1$. Let $v \in V_{X} \backslash\{1\}$; as $(G, V)$ satisfies $\mathcal{N}_{q}$, there exists $Q \in \operatorname{Syl}_{q}(G)$ with $Q \unlhd \mathbf{C}_{G}(v)$. Since $X \leq \mathbf{C}_{K}(v)$ and $\left[Q, \mathbf{C}_{K}(v)\right] \leq$ $Q \cap K=1$, we see that $Q \leq Y$. So $Q \unlhd \mathbf{C}_{G}(v) \cap Y=\mathbf{C}_{Y}(v)$. Observe also that $q$ divides $\left|Y / \mathbf{C}_{Y}\left(V_{X}\right)\right|$, because otherwise $\mathbf{C}_{G}\left(V_{X}\right)$ would contain a Sylow $q$-subgroup of $G$, against one of the assumptions. Hence, $\left(Y, V_{X}\right)$ satisfies $\mathcal{N}_{q}$.

We remark that the proof of the next result depends, via [12, Theorem 2.2 and Theorem 2.3], on the classification of finite simple groups. The classification is also used in Propostion 2.8 and Proposition 2.9. 
Theorem 2.5. Let $(G, V)$ satisfy $\mathcal{N}_{q}$ and assume that $(|V|,|G|)=1$. Then $G / \mathbf{C}_{G}(V)$ is a subgroup of $\Gamma(V)$.

Proof. Let $G$ be a counterexample of minimal order. Hence, $\mathbf{C}_{G}(V)=1$. Let $Q$ be a Sylow $q$-subgroup of $G$. Write $|V|=r^{a}$ and $\left|\mathbf{C}_{V}(Q)\right|=r^{b}$, where $r$ is a suitable prime obviously different from $q$.

By part (b) of Theorem 2.2, $G$ is non-solvable; in particular, by the Odd Order Theorem, we have $r \neq 2$.

Now, Claim (a) of Proposition 2.3 yields $q \neq 2$ as well, whereas Claim (d) ensures the existence of a primitive prime divisor $t \in \pi(G)$ of $(r, a)$. We remark that $a$ divides $t-1$, so $a+1 \leq t$. In what follows, we will denote by $T$ a subgroup of order $t$ of $G$.

Let $K \unlhd G$ such that $G / K$ is simple. If $q \in \pi(K)$, then $(K, V)$ satisfies $\mathcal{N}_{q}$, and the minimality of $G$ yields $K \leq \Gamma(V)$. This in turn implies the solvability of $G$ via part (b) of Theorem 2.2, and this is not the case. Therefore, $q$ does not divide $|K|$ and $G=\mathbf{O}^{q^{\prime}}(G)$.

If $G / K$ is abelian, then $K$ is a normal $q$-complement of $G$ and hence $G$ would be solvable by part (a) of Theorem 2.2 , a contradiction.

Thus $G / K$ is a non-abelian simple group; this being true for every maximal normal subgroup $K$ of $G$, it follows that $G$ is perfect. This implies, in particular, that every cyclic normal section of $G$ is central in $G$.

Let $T \in \operatorname{Syl}_{t}(G)$ and $T_{0}=T \cap K$. Then $G=K \mathbf{N}_{G}\left(T_{0}\right)=K \mathbf{C}_{G}\left(T_{0}\right)$, because $\operatorname{Aut}\left(T_{0}\right)$ is abelian. But if $T_{0} \neq 1$, then $\mathbf{C}_{G}\left(T_{0}\right)$ is cyclic (being isomorphic to a subgroup of the multiplicative group of a finite field, as $V$ is an irreducible $T_{0^{-}}$ module) and hence $G=K$, a contradiction. So $T$ acts coprimely on $K$ and, for every $p \in \pi(K)$, there is a $T$-invariant Sylow $p$-subgroup $P$ of $K$. By [2, Lemma 6], if $p$ is odd then $T$ centralizes $P$ and hence $K=Q \mathbf{C}_{K}(T)$, where $Q$ is a $T$-invariant Sylow 2-subgroup of $K$. Hence, $\left[T^{g}, K\right] \leq \mathbf{O}_{2}(K)$ for all $g \in G$. As $T^{G} K=G$ and $\mathbf{O}^{q^{\prime}}(G)=G$, it follows $T^{G}=G$ and hence $K / \mathbf{O}_{2}(K)$ is central in $G / \mathbf{O}_{2}(K)$. Therefore, $G / \mathbf{O}_{2}(K)$ is a quasi-simple group.

Consider now the "iterated commutator subgroup" $E_{j}=[K, G, \cdots, G]$, where $G$ appears $j$ times; observe that, by the paragraph above, $E_{j}$ lies in $\mathbf{O}_{2}(K)$ for every $j \geq 1$ (note also that $E_{j}$ is clearly normal in $G$ ). Now, let $j_{0}$ be the smallest positive integer such that $E_{j_{0}}=E_{j_{0}+1}$, and set $E:=E_{j_{0}}$. Taking into account that $G$ is perfect, Theorem 4.22 of [11] yields that $K / E$ is central in $G / E$, so $G / E$ is quasi-simple. In particular, since Theorems 2.2 and 2.3 of [12] show that $G$ itself cannot be quasi-simple under our assumptions, we have $E>1$.

We claim that $E$ is an extraspecial 2-group. In fact, let $A$ be an abelian characteristic subgroup of $E$. Then $A T \leq G$ acts irreducibly on $V$, because the order of $T$ is a primitive prime divisor of $(r, a)$; moreover, $V$ viewed as a module for $A$ is homogeneous, as otherwise it would have $t$ homogeneous components, against the fact that $a=\operatorname{dim}(V)<t$. As a consequence $A$ is cyclic, so we have established that every abelian characteristic subgroup of $E$ is cyclic. In this situation, Theorem 1.2 of [17] yields that $E=E_{0} W$, where either $E_{0}$ is extraspecial or of order 2, whereas $W$ has a cyclic subgroup $U$ that is characteristic in $E$ and of index at most 2 in $W$; moreover, $E_{0} U$ is also a characteristic subgroup of $E$. Now, the fact that $[E, G]=E$ together with the fact that every cyclic normal section of $G$ is central in $G$, easily implies that $W=1$. For the same reason, $E$ cannot be cyclic (since $E \neq 1$ ), and therefore $E$ is an extraspecial 2-group, as claimed. 
Write $|E / \mathbf{Z}(E)|=2^{2 n}$. By [17, Corollary 2.6], we have that $2^{n}$ divides $a=$ $\operatorname{dim}(V)$. Also, observe that $E_{1}=[E, T] \neq 1$ (because the centralizer of $T$ in $\mathrm{GL}(V)$ is cyclic and $E$ is not) and that (see for instance [5, Lemma 2.2]) $E_{1}$ is extraspecial (say of order $2^{2 m+1}$ ), such that $T$ acts fixed-point freely on $E_{1} / \mathbf{Z}\left(E_{1}\right)$. By Lemma 2.4(i) in [6], we get $t \leq 2^{m}+1 \leq 2^{n}+1 \leq a+1 \leq t$. We conclude that $t=2^{n}+1=a+1$, and therefore $a=2^{n}$.

Note that $E \not Q_{8}$, as $\mathbf{N}_{G}(E) / \mathbf{C}_{G}(E)$ is non-solvable. Let $x$ be a non-central involution of $E$; let $X=\langle x\rangle$ and $Y=\mathbf{C}_{G}(X)$. As $x \notin \mathbf{Z}(E)$, then $\operatorname{dim}\left(V_{X}\right)=a / 2$ : in fact, the dimension of the fixed-point space of $X$ in $V$ is invariant by extensions of the ground field, and it is equal to $\left[\chi_{X}, 1_{X}\right]=\chi(1) / 2=a / 2$, because $\chi(x)=0$, where $\chi$ is the (Brauer, but also ordinary as $r \neq 2$ ) character corresponding to $V$ on a suitable extension of $\mathrm{GF}(r)$.

So, by (c) of Proposition 2.3, $\left|V_{X}\right|>\left|V_{Q}\right|$ and this yields that $q$ divides $\mid G$ : $\mathbf{C}_{G}\left(V_{X}\right) \mid$. Hence $\left(Y, V_{X}\right)$ satisfies $\mathcal{N}_{q}$ by Lemma 2.4. As $Y<G$, by minimality $Y / \mathbf{C}_{Y}\left(V_{X}\right) \leq \Gamma\left(V_{X}\right)$ and part (a) of Lemma 2.1 gives that $q$ divides $\operatorname{dim}\left(V_{X}\right)=a / 2$ which is a power of 2 , against $q \neq 2$.

Proposition 2.6. Let $S \simeq \mathrm{PSL}_{2}\left(p^{a}\right)$ or $S \simeq \mathrm{SL}_{2}\left(p^{a}\right)$, with $p$ prime and $a \geq 1$. Let $\pi_{+}=\pi\left(p^{a}+1\right)$ and $\pi_{-}=\pi\left(p^{a}-1\right)$. For a subset $\pi$ of vertices of $\Delta(S)$, we denote by $\Delta_{\pi}$ the subgraph of $\Delta=\Delta(S)$ induced by the subset $\pi$. Then

(a) if $p=2, \Delta(S)$ has three connected components, $\{p\}, \Delta_{\pi_{+}}$and $\Delta_{\pi_{-}}$, and each of them is a complete graph.

(b) if $p>2$ and $p^{a}>5$, then $\Delta(S)$ has two connected components, $\{p\}$ and $\Delta_{\pi_{+} \cup \pi_{-}}$; moreover, both $\Delta_{\pi_{+}}$and $\Delta_{\pi_{-}}$are complete graphs, no vertex $\neq 2$ in $\pi_{+}$is adjacent to any vertex $\neq 2$ in $\pi_{-}$and 2 is adjacent to all other vertices in $\Delta_{\pi_{+} \cup \pi_{-}}$.

Proof. It is well-known (see for instance [7, Theorems 38.1, 38.2]) that, for $a \geq 2$

$$
\operatorname{cd}\left(\mathrm{SL}_{2}\left(2^{a}\right)\right)=\operatorname{cd}\left(\mathrm{PSL}_{2}\left(2^{a}\right)\right)=\left\{1,2^{a}-1,2^{a}, 2^{a}+1\right\}
$$

and that for $p \neq 2$ and $p^{a}>5$

$$
\begin{gathered}
\operatorname{cd}\left(\mathrm{PSL}_{2}\left(p^{a}\right)\right)=\left\{1, p^{a}-1, p^{a}, p^{a}+1, \frac{1}{2}\left(p^{a}+\epsilon\right)\right\} \text { where } \epsilon=(-1)^{\frac{p^{a}-1}{2}} \\
\operatorname{cd}\left(\mathrm{SL}_{2}\left(p^{a}\right)\right)=\left\{1, p^{a}-1, p^{a}, p^{a}+1, \frac{1}{2}\left(p^{a}+\epsilon\right)\right\} \text { where } \epsilon= \pm 1
\end{gathered}
$$

$\left(\right.$ while $\operatorname{cd}\left(\mathrm{PSL}_{2}(5)\right)=\{1,3,4,5\}$ and $\left.\operatorname{cd}\left(\mathrm{SL}_{2}(5)\right)=\{1,2,3,4,5,6\}\right)$.

Proposition 2.7. Let $G$ be an almost-simple group with socle $S \simeq \operatorname{PSL}_{2}\left(p^{a}\right)$, where $p$ is a prime. If $s$ is a prime divisor of $|G / S|$, then $s$ is adjacent in $\Delta(G)$ to every prime in $\pi\left(p^{2 a}-1\right)$.

Proof. This follows from Theorem A of [20].

Proposition 2.8. Let $G$ be an almost-simple group with socle $S$ and let $\sigma=\pi(S)$ be the set of prime divisors of the order of $S$. Then

(a) $G / S$ has a central cyclic $\sigma$-complement $A / S$ and $A / S$ has a regular orbit on $\operatorname{Irr}(S)$.

(b) If $q$ and $s$ are distinct non-adjacent vertices of $\Delta(G)$, then $G$ does not have abelian Hall $\{q, s\}$-subgroups and $|\{q, s\} \cap \sigma| \geq 1$. If $s \notin \sigma$, then $S$ is a simple group of Lie Type in characteristic $q$. 
Proof. (a) The first assertion follows by [18, Lemma 2.10]) and the second by [18, Proposition 2.6],

(b) By part (a) we may assume that, say, $q \in \pi(S)$. If $s \notin \pi(S)$, both conclusions follow form [18, Theorem 2.7]; if $s \in \pi(S)$, one applies [18, Lemma 2.8].

Proposition 2.9. Let $G$ be an almost-simple group with socle $S$ and let $\pi \subseteq \mathrm{V}(G)$ with $|\pi|=3$. Then the subgraph of $\Delta(G)$ induced by $\pi$ is empty if and only if $\pi \cap \pi(G / S)=\emptyset, S \simeq \mathrm{PSL}_{2}\left(p^{a}\right)$ where $p$ is a prime, $a$ is a positive integer, $\pi=$ $\{p, q, s\}, q$ divides $p^{a}+1, s$ divides $p^{a}-1$ and $q, s \neq 2$.

Proof. By Proposition 2.6, one readily sees that the above conditions are sufficient for the subgraph of $\Delta(G)$ induced on $\pi$ to be an empty triangle.

We now prove the other implication. By Proposition $2.8,|\pi \cap \pi(S)| \geq 2$. If say, $s \notin \pi(S)$ then by Proposition 2.8, $S$ is a finite simple group of Lie type both in characteristic $p$ and $q$. As the only examples of double characteristic are $\mathrm{PSL}_{2}(4) \simeq$ $\mathrm{PSL}_{2}(5), \mathrm{PSL}_{2}(7) \simeq \mathrm{PSL}_{3}(2)$ and $\mathrm{PSU}_{4}(2) \simeq \mathrm{PSp}_{4}(3)$ ), we get a contradiction by observing that the outer automorphism groups of those groups have order 2. Hence, $\pi \subseteq \pi(S)$ and then $S \simeq \operatorname{PSL}_{2}\left(p^{a}\right)$ for a prime $p$ and a positive integer $a$, by [18, Lemma 2.9]. By Proposition 2.7, no prime in $\pi$ can divide $|G / S|$. Observing that in the degree graph $\Delta\left(\mathrm{PSL}_{2}\left(p^{a}\right)\right)$ the vertex $p$ (the characteristic) is an isolated vertex, that both $\pi\left(p^{a}+1\right)$ and $\pi\left(p^{a}-1\right)$ induce complete subgraphs and that if $p \neq 2$ then 2 is adjacent to all vertices distinct from $p$, the conditions $q, s \neq 2$ and (say) $q \in \pi\left(p^{a}+1\right), s \in \pi\left(p^{a}-1\right)$ are immediate.

Proposition 2.10. Let $G$ be a finite group, $M$ a non-abelian minimal normal subgroup of $G$ and $C=\mathbf{C}_{G}(M)$. Then the following conclusions hold.

(a) If $q$ is a prime divisor of $|G / M C|$ and $q$ does not divide $|M|$, then there exists $\theta \in \operatorname{Irr}(M)$ such that $q$ divides $\left|G: I_{G}(\theta)\right|$.

(b) If $q$ is a prime divisor of $|G / C|$, then there exists $\theta \in \operatorname{Irr}(M)$ such that $q$ divides $\chi(1)$ for all $\chi \in \operatorname{Irr}(G \mid \theta)$.

(c) If $M$ is not a simple group, then $\Delta(G / C)$ is a complete graph.

Proof. We first consider Claim (a). Write $M=S_{1} \times \cdots \times S_{k}$, where the groups $S_{i}$ are isomorphic to a suitable non-abelian simple group $S$, and $k \geq 1$. Set $K=$ $\bigcap_{i=1}^{k} \mathbf{N}_{G}\left(S_{i}\right)$, so $K$ is the kernel of the permutation action of $G$ on the set $\Omega=$ $\left\{S_{1}, \ldots, S_{k}\right\}$. Let $q \in \pi(K) \backslash \pi(S)$. By Proposition 2.8, Out $(S)$ has a cylic normal (central) Sylow $q$-subgroup, and hence $K / M$ has a normal Sylow $q$-subgroup $Q_{0}$. If $Q_{0} \neq 1$, then $Q_{0}$ does not act trivially on the set of the conjugacy classes of $M$. Hence, there exists $\theta \in \operatorname{Irr}(M)$ such that $q$ divides $\left|G: I_{G}(\theta)\right|$. If $Q_{0}$ is trivial, then $q$ divides $|G / K|$ and we can apply (a consequence of) Lemma 8 of [3]: there exist disjoint subsets $\Gamma_{1}, \Gamma_{2}$ of $\Omega$ such that $q$ divides the index of the intersection $I$ of the setwise stabilizers of $\Gamma_{1}$ and $\Gamma_{2}$ in $G$. Given non-principal irreducible characters $\phi_{1}, \phi_{2}$ of $S$ with distinct degrees, consider $\theta \in \operatorname{Irr}(M)$ which is the product of irreducible characters $\theta_{i}$ of $S_{i}$ such that $\theta_{i}(1)=\phi_{1}(1)$ if $S_{i} \in \Gamma_{1}, \theta_{i}(1)=\phi_{2}(1)$ if $S_{i} \in \Gamma_{2}$ and $\theta_{i}=1_{S_{i}}$ otherwise. One easily checks that $I_{G}(\theta)=I$ and this finishes the proof of (a).

Claim (b) is clear if $q \in \pi(M)$, and it follows from (a) if $q \notin \pi(M)$. As for (c), this is the Main Theorem of [16].

We are now ready to prove Theorem A. 
Proof of Theorem A. We first observe that, by [10, Corollary 11.29], the $\pi$-parts of the irreducible character degrees of $G$ and of $\mathbf{O}^{\pi^{\prime}}(G)$ are the same. Thus, we can henceforth assume that $G=\mathbf{O}^{\pi^{\prime}}(G)$.

The sufficiency of the condition follows from Proposition 2.9. We now start proving the converse. We will first establish the following claim:

$G$ has a composition factor $S \simeq \mathrm{PSL}_{2}\left(p^{a}\right)$ such that $\pi \subseteq \pi(S)$.

Let $G$ be a minimal counterexample to the above claim, and let $N$ be a non-trivial normal subgroup of $G$. If $\pi \subseteq \mathrm{V}(G / N)$, then $G$ would not be a counterexample. So, for every minimal normal subgroup $N$ of $G$ there exists a prime $t \in \pi$ such $G / N$ has an abelian normal Sylow $t$-subgroup.

As the first step, we show that $G$ has a unique minimal normal subgroup. In order to do this, we first prove that if $N$ is minimal normal in $G, t \in \pi, t \notin \mathrm{V}(G / N)$, then there exists $\theta \in \operatorname{Irr}(N)$ such that for every $\chi \in \operatorname{Irr}(G \mid \theta)$ the prime $t$ divides the degree $\chi(1)$. This is certainly true if $t \in \pi(N)$ and $N$ is nonabelian (just take any $\theta \in \operatorname{Irr}(N)$ with $t \mid \theta(1))$. Let $T \in \operatorname{Syl}_{t}(G), t \in \pi$, such that $T N / N$ is abelian and normal in $G / N$. Assume next that $N$ is nonabelian and that $t \notin \pi(N)$. Since $t \in \mathrm{V}(G)$, we have $[T, N] \neq 1$ and hence by coprimality there is (a conjugacy class of $N$ and thus) an irreducible character $\theta$ of $N$, that is not invariant in $T N$. By Clifford Theory, then $t \mid \chi(1)$ for all $\chi \in \operatorname{Irr}(G \mid \theta)$. Finally, assume that $N$ is abelian, $|N|=r^{k}, r$ a prime. If $r \neq t$, then $\mathbf{C}_{N}(T)=1$ and (by coprimality), $t$ divides $\left[T N: I_{T N}(\theta)\right]$ for every non-principal $\theta \in \operatorname{Irr}(N)$. If $N$ is a $t$-group, then $T \unlhd G$ and $N=T^{\prime}$. So, again, if $\theta \in \operatorname{Irr}(N)$ is non-principal, then $t$ divides $\chi(1)$ for every $\chi \in \operatorname{Irr}(G \mid \theta)$.

Now, if $N_{1}$ and $N_{2}$ are distinct minimal normal subgroups of $G$ and $t_{i} \in \pi$ are such that $t_{i} \notin \mathrm{V}\left(G / N_{i}\right)$ for $i=1,2$, then we observe that $t_{1} \neq t_{2}$; otherwise $G$ would have an abelian normal Sylow $t$-subgroup for $t=t_{1}=t_{2}$. By the previous paragraph, there are characters $\theta_{i} \in \operatorname{Irr}\left(N_{i}\right)$ such that $t_{i}$ divides the degree of every irreducible character of $G$ lying over $\theta_{i}$, for $i=1,2$. Hence, the product $t_{1} t_{2}$ divides the degree of every $\chi \in \operatorname{Irr}(G)$ lying above $\theta_{1} \times \theta_{2} \in \operatorname{Irr}\left(N_{1} \times N_{2}\right)$, a contradiction. Therefore, there exists an unique minimal normal subgroup $N$ of $G$.

If $N$ is nonabelian, then $\mathbf{C}_{G}(N)=1$ and by part (c) of Proposition 2.10, $N$ is simple. Hence $G$ is almost-simple with socle $N$ and we conclude by Proposition 2.9.

We will next derive a contradiction, under the assumption that $N$ is abelian and that $\pi \nsubseteq \mathrm{V}(G / N)$. Let then $N$ be elementary abelian of order $r^{k}$, where $r$ is a suitable prime, and let $p \in \pi$ such that $p \notin \mathrm{V}(G / N)$. Let $P$ be a Sylow $p$-subgroup of $G$. We have two cases:

(I) $r \neq p$; in this case $N$ is not contained in the Frattini subgroup $\boldsymbol{\Phi}(G)$, as otherwise $G$ would have a normal abelian Sylow $p$-subgroup, a contradiction. So, there exists a complement $H$ of $N$ in $G$ and hence every $\theta \in \operatorname{Irr}(N)$ extends to $I_{G}(\theta)$. Since $N=[P, N], p$ divides $\left[H: I_{H}(\theta)\right]$, for every non-principal $\theta \in \operatorname{Irr}(N)$. We remark that in this case $\mathbf{C}_{H}(N)=1$, as $N=\mathbf{F}(G)$.

(II) $N \leq P$, where $P$ is a Sylow $p$-subgroup of $G$; so $P \unlhd G$ and $N=P^{\prime}$. Let $H$ be a $p$-complement of $G$. $H$ acts faithfully on $P=\mathbf{F}(G)$ and hence also on $P / N$, because $N \leq \boldsymbol{\Phi}(P)$. By coprimality, for every $\theta \in \operatorname{Irr}(N)$ there exists $\psi \in \operatorname{Irr}(P \mid \theta)$ such that $I_{H}(\psi)=I_{H}(\theta)$ and $\psi$ extends to $I_{G}(\psi)=P I_{H}(\theta)$. If $\theta$ is non-principal, then $p$ divides $\psi(1)$. 
Write $\pi=\{p, q, s\}$. By Gallagher's theorem, in both cases (I) and (II) the inertia subgroup $I_{H}(\theta)$, for every non-principal $\theta \in \operatorname{Irr}(N)$, contains a Hall $\{q, s\}$ subgroup $L$ of $H, L \unlhd I_{H}(\theta)$ and $L$ is abelian. So, in particular, $H$ has abelian Hall $\{q, s\}$-subgroups.

We now consider the cases (I) and (II) separately.

(I): Assume $r \neq p$, so the complement $H$ of $N$ in $G$ acts faithfully on $N$. Let $B$ be the solvable radical of $H$. Note that if $q$ divides $|B|$, then $(B, N)$ satisfies $\mathcal{N}_{q}$ and hence part (b) of Theorem 2.2 yields the solvability of $H$, a contradiction. Arguing similarly for the prime $s$, we deduce that $B$ is a $\{q, s\}^{\prime}$-group. Hence, $\{q, s\} \in \mathrm{V}(H / B)=\pi(H / B)$. Let $A / B$ be a chief factor of $H / B$ and let $C / B=$ $\mathbf{C}_{H / B}(A / B)$. Note that $\{q, s\} \cap \pi(H / C)$ cannot be empty, since $p \notin \pi(G / C)$ because $H \simeq G / N$ has a normal Sylow $p$-subgroup, and $\mathrm{O}^{\pi^{\prime}}(H)=H$. On the other hand, if $\{q, s\} \subseteq \pi(H / C)$ then $A / B$ is simple by part (c) of Proposition 2.10, thus $H / C$ is almost simple. But Lemma 2.8 yields that $H / C$ has no abelian Hall $\{q, s\}$ subgroups, again a contradiction. We conclude that, $q \in \pi(H / C)$ and $s \notin \pi(H / C)$. Then, by Proposition 2.10(b), there exists $\theta \in \operatorname{Irr}(A / B)$ such that $q \mid \chi(1)$ for all $\chi \in \operatorname{Irr}(H / B)$ lying over $\theta$; moreover, since $s \in \mathrm{V}(C / B)=\pi(C / B)$ (because $C / B$ has no non-trivial abelian normal subgroups), there exists $\psi \in \operatorname{Irr}(C)$ such that $s \mid \psi(1)$. So, every irreducible character of $H / B$ lying over $\theta \times \psi \in \operatorname{Irr}(A / B \times C / B)$ has a degree divisible by $q s$, the final contradiction which rules out (I).

(II) Assume now $N=P^{\prime}$; so $P \unlhd G$, and we can consider a complement $H$ of $P$ in $G$. Let $L=\mathbf{C}_{H}(N)$. Note that $L$ has abelian normal Sylow $q$-subgroups and Sylow $s$-subgroups, because $I_{H}(\lambda)$ has this property if $\lambda$ is any non-principal character in $\operatorname{Irr}(N)$, and $L \leq I_{H}(\lambda)$. So either $q$ or $s$ does not divide $|L|$, because the abelian normal Hall $\{q, s\}$-subgroup of $L$ has a regular orbit on $\operatorname{Irr}(P / N)$. Say, $(s,|L|)=1$. As a consequence, we get $s|| H / L \mid$, so $(H, N)$ satisfies $\mathcal{N}_{s}$ and Theorem 2.5 yields $H / L \leq \Gamma(N)$. Thus, by Lemma $2.1 H$ has a normal $s$-complement and $s$ divides $|H / L: \mathbf{F}(H / L)|$. If $q$ divides $|H / L|$ as well, the same argument yields that $q$ divides $|H / L: \mathbf{F}(H / L)|$. But [17, Proposition 17.3] implies now that $q$ and $s$ are adjacent vertices in $\Delta(H / L)$, thus in $\Delta(G)$, against the assumptions.

Our conclusion so far is that $q \notin \pi(H / L)$. So, let $1 \neq Q \leq L$ be a Sylow $q$ subgroup of $H$; recall that $Q$ is normal in $L$, thus normal in $H$. Let $V=P / N$; so $V=[V, Q] \times \mathbf{C}_{V}(Q)$. If $1 \neq \alpha \in \operatorname{Irr}([V, Q])$ and $\beta \in \operatorname{Irr}\left(\mathbf{C}_{V}(Q)\right)$, then $I_{H}(\alpha \times \beta)=$ $I_{H}(\alpha) \cap I_{H}(\beta)$ contains a unique Sylow $s$-subgroup $S$ of $H$. By letting $\alpha$ fixed and varying $\beta$, we deduce that $S$ centralizes $\mathbf{C}_{V}(Q)$. As $\mathbf{O}^{\pi^{\prime}}(H)=H$, we conclude that $H$ centralizes $\mathbf{C}_{V}(Q)$, so, writing $[V, Q]=P_{0} / N$, we have that $P_{0} H \unlhd G$. Note that $P_{0}$ is non-abelian, as otherwise by coprime action $P_{0}=\left[P_{0}, Q\right] \times N$ and $[P, Q]=[P, Q, Q]$ hence (as $\left.[P, Q] \leq P_{0}\right)\left[P_{0}, Q\right]=[P, Q] \unlhd G$, against the fact that $N$ is the only minimal normal subgroup of $G$. We deduce that $\pi \subseteq \mathrm{V}\left(P_{0} H\right)$ and this yields $P_{0}=P$, because of the minimality of $G$. Hence, $V$ is a faithful $H$-module and $(H, V)$ satisfies $\mathcal{N}_{s}$. By applying part (a) of Theorem 2.2 (or, alternatively, Theorem 2.5 as the action is coprime), we get the final contradiction which rules out (II) as well.

The claim in the second paragraph of this proof is now established. Let $A / B$ be a non-abelian chief factor of $G$ with $A / B \simeq T^{k}, T=\mathrm{PSL}_{2}\left(p^{a}\right)$ with $\pi \subseteq \pi(T)$. Clearly, then $k=1$. Moreover, Proposition 2.6 yields $\pi=\{p, q, s\}$ where $q$ divides 
$p^{a}+1, s$ divides $p^{a}-1$ and $q, s \neq 2$. Note that, in particular, $p^{a}$ is neither a Mersenne nor a Fermat prime and $p^{a} \neq 2,9$.

Next, we prove that $G$ has a unique non-abelian composition factor of order divisible by some prime in $\pi$. Assume that there is, in a chief series containing $A$ and $B$, another non-abelian chief factor $C / D$ of $G$ with $\pi \cap \pi(C / D) \neq \emptyset$. If $A \leq D$, then $\mathbf{C}_{G / B}(A / B)$ has a normal section isomorphic to $C / D$ (since $\operatorname{Out}(A / B)$ is solvable) and hence we get an edge between two primes in $\pi$ in the subgraph $\Delta\left(A / B \times \mathbf{C}_{G / B}(A / B)\right)$ of $\Delta(G)$, a contradiction. If $C \leq B$, then similarly (using also Proposition $2.10(\mathrm{c})$ ) we get that $\mathbf{C}_{G / D}(C / D)$ has a normal section isomorphic to $A / B$ and we get a contradiction in a similar way.

So, there exist subgroups $A, B \unlhd G$, with $A=A^{\prime}$ (i.e. $A$ perfect) such that $A / B \simeq T=\mathrm{PSL}_{2}\left(p^{a}\right)$ with $\pi \subseteq \pi(T)$ and both $B$ and $G / A \pi$-solvable. Let $C=\mathbf{C}_{G}(A / B)$; so $G / C$ is almost simple with socle isomorphic to $T$ and by Proposition 2.7 every prime divisor of $|G / A C|$ is adjacent in $\Delta(G)$ to all the primes in both $\pi\left(p^{a}-1\right)$ and $\pi\left(p^{a}+1\right)$. Therefore, $G / A C$ is a $\pi^{\prime}$-group. As $\mathbf{O}^{\pi^{\prime}}(G)=G$, we get $A C=G$. So, $G / B=A / B \times C / B$ and this implies that $\pi \cap \mathrm{V}(C / B)=\emptyset$, so $C / B$ has a normal abelian Hall $\pi$-subgroup $D / B$; again, the assumption $\mathbf{O}^{\pi^{\prime}}(G)=G$ yields $C=D$.

In order to complete the proof, we can assume that $G=A$ is perfect. In fact, $A$ certainly satisfies the hypotheses of this theorem, so, if $A<G$, induction yields that $A$ is isomorphic either to $\mathrm{PSL}_{2}\left(p^{a}\right)$ or to $\mathrm{SL}_{2}\left(p^{a}\right)$. Now, if $2 \in \pi$ (which implies $2=p$ ), then $B=1$, and we get $G=A \times D$; on the other hand, if $2 \notin \pi$ and $D_{0}$ is the Hall $2^{\prime}$-subgroup of $D$, it can be easily checked that $G=A \times D_{0}$. In any case, the conclusion of this theorem is satisfied by $G$ and we are done.

Note that we can also assume that $B>1$, as $G / B \simeq \operatorname{PSL}_{2}\left(p^{a}\right)$. We will show that then $G \simeq \mathrm{SL}_{2}\left(p^{a}\right)$, with $p \neq 2$. Let $M \leq B$ be such that $B / M$ is a chief factor of $G$.

Assume first that $M=1$. It is enough to show that $B \leq \mathbf{Z}(G)$ : as $G$ is perfect, then $G$ will be the Schur representation group of $G / B$ and hence $G \simeq \operatorname{SL}_{2}\left(p^{a}\right)$.

In this situation $B$ is a minimal normal subgroup of $G$, which is in fact (elementary) abelian; otherwise $B$ would be a non-abelian simple group by Proposition $2.10(\mathrm{c})$, whence $G / B \mathbf{C}_{G}(B)$ would be solvable and we would have $G=$ $B \times \mathbf{C}_{G}(B)$ because $G$ is perfect; but this gives $\mathbf{O}^{\pi^{\prime}}(G)<G$, a contradiction. Let us assume, working by contradiction, that $B$ is non-central in $G$. Thus, $\mathbf{C}_{B}(G / B)=1$

Then let $\lambda$ be any non-principal character in $\operatorname{Irr}(B)$. Setting $I=I_{G}(\lambda)$, we see that $I<G$, so $I / B$ is isomorphic to a proper subgroup of $\operatorname{PSL}_{2}\left(p^{a}\right)$. Recall that the subgroups of $\mathrm{PSL}_{2}\left(p^{a}\right)$ are of the following types (see [8, II.8.27] or [21, Chapter 3 , Theorem 6.25]), where $d=\left(2, p^{a}-1\right)$ : (i) dihedral groups of order $\left(2\left(p^{a} \pm 1\right)\right) / d$ and their subgroups; (ii) semidirect products of elementary abelian groups of order $p^{a}$ by cyclic groups of order $\left(p^{a}-1\right) / d$ and their subgroups; (iii) $A_{4}$ (unless $p=2$ and $a$ is odd $) ; S_{4}\left(\right.$ when $\left.p^{2 a} \equiv 1(\bmod 16)\right) ; A_{5}\left(\right.$ when $\left.p^{a}\left(p^{2 a}-1\right) \equiv 0(\bmod 5)\right)$; (iv) $\mathrm{PSL}_{2}\left(p^{b}\right)$ or $\mathrm{PGL}_{2}\left(p^{b}\right)$, where $b$ divides $a$ (for $p \neq 2, \mathrm{PSL}_{2}\left(p^{a}\right)$ has a subgroup isomorphic to $\mathrm{PGL}_{2}\left(p^{b}\right)$ if and only if $a / b$ is even).

Considering the indices of the above listed subgroups, and recalling that $|G: I|$ cannot be a multiple of more than one prime in $\pi$ (and also that the characteristic $p$ is in $\pi$ and that if $p \neq 2$ then all primes in $\pi$ are odd), we can rule out that $I / B$ is of type (i). 
As for type (iii), if $I / B \simeq A_{4}$ or $I / B \simeq S_{4}$, then we get $p=2$ and (say) $q=3$. In the latter case, (as 2 cannot divide $|G: I|)$, we must have $G / B \simeq \operatorname{PSL}_{2}\left(2^{3}\right)$, but $\mathrm{PSL}_{2}\left(2^{3}\right)$ does not have subgroups isomorphic to $S_{4}$.

If $I / B \simeq A_{4}$, then the Schur representation group of $I / B$ is a $\{2,3\}$-group. If $\operatorname{Irr}(I \mid \lambda)$ contains a linear character, then Gallagher's theorem yields that there exists $\psi \in \operatorname{Irr}(I \mid \lambda)$ such that $\psi(1)=3$; on the other hand, any nonlinear character in $\operatorname{Irr}(I \mid \lambda)$ has a degree divisible by 2 or 3 . In any case, we get a contradiction.

In order to rule out type (iii), it remains to consider the case $I / B \simeq A_{5}$. In this situation, certainly $p$ divides $|G: I|$, therefore we have $\{q, s\}=\{3,5\}$; it will be enough to show that $\operatorname{Irr}(I \mid \lambda)$ contains a character of degree divisible by 3 (to this end, we can assume that $\lambda$ does not extend to $I$ ). Set $B_{0}=\operatorname{ker}(\lambda)$, and observe that $I / B_{0}$ does not split over $B / B_{0}$, as otherwise $\lambda$ would have an extension to $I$; moreover, $B / B_{0}$ is central in $I / B_{0}$. As a consequence, we get $I / B_{0} \simeq \mathrm{SL}_{2}(5)$. Now, the characters in $\operatorname{Irr}(I \mid \lambda)$ are precisely the faithful characters of $I / B_{0}$; among those, there is a character of degree 6 , as wanted.

Consider now type (iv): $I / B \simeq \mathrm{PSL}_{2}\left(p^{b}\right)$ or $\mathrm{PGL}_{2}\left(p^{b}\right)$, where $b$ divides $a$ (note that we already considered the case $p^{b} \in\{4,5\}$, i.e., $I / B \simeq A_{5}$ ). Clearly $p$ divides $|G: I|$, therefore we have (say) $q \in \pi\left(p^{b}+1\right)$ and $s \in \pi\left(p^{b}-1\right)$. If $p^{b}=9$, then $I / B \simeq \mathrm{PSL}_{2}(9) \simeq A_{6}$ or $\mathrm{PGL}_{2}(9)$, and we have $p=3$; but since both $q$ and $s$ cannot appear in $|G: I|$, one of them should be 2 , which is impossible. So we can assume $p^{b} \neq 9$. Let $I_{0}$ be the normal subgroup of $I$ (with $\left|I: I_{0}\right| \in\{1,2\}$ ) such that $I_{0} / B \simeq \mathrm{PSL}_{2}\left(p^{b}\right)$ and let $B_{0}=\operatorname{ker}(\lambda)$. If $I_{0} / B_{0}$ does not split over $B / B_{0}$, then $B / B_{0} \leq\left(I_{0} / B_{0}\right)^{\prime}$ and, as $B / B_{0} \leq \mathbf{Z}\left(I_{0} / B_{0}\right)$ as well, we deduce that $I_{0} / B_{0} \simeq \mathrm{SL}_{2}\left(p^{b}\right)$, the representation group of $I_{0} / B$. But $\mathrm{SL}_{2}\left(p^{b}\right)$ has faithful characters of degree $p^{b}+1$ (as well as $p^{b}-1$ ), and hence there exists $\theta \in \operatorname{Irr}\left(I_{0} \mid \lambda\right.$ ) such that $q$ divides $\theta(1)$. If $I_{0} / B_{0}$ splits over $B / B_{0}$, then one gets the same conclusion by Gallagher's theorem. Therefore, there exists $\psi \in \operatorname{Irr}(I \mid \lambda)$ such that $q$ divides $\psi(1)$ and hence a $\chi \in \operatorname{Irr}(G \mid \lambda)$ such that $p q$ divides $\chi(1)$, a contradiction.

Finally, assume that $I / B$ is of type (ii); then (say) $q \in \pi\left(p^{a}+1\right)$ divides $|G: I|$. Observe that $I / B$ is a Frobenius group with kernel $P / B$ (a Sylow $p$-subgroup of $G / B)$ and cyclic complement $C / B$. Moreover, $s$ divides $|C / B|$. Now, since $I / B$ has irreducible characters of degree divisible by $s$, we get that $\lambda$ does not extend to $I$. Therefore, as every Sylow subgroup of $I / B$ other than $P / B$ is cyclic, [10, (11.31)] yields that $\lambda$ does not extend to $P$. As a consequence, if $\beta \in \operatorname{Irr}(I \mid \lambda)$, then $p$ divides $\beta(1)$ and $p q$ divides the degree of $\beta^{G} \in \operatorname{Irr}(G)$, a contradiction. Therefore, $B$ is central in $G$ and hence $G \simeq \mathrm{SL}_{2}\left(p^{a}\right)$.

Assume finally, working by contradiction, that $M>1$ : then induction yields that $G / M \simeq \mathrm{SL}_{2}\left(p^{a}\right), p \neq 2$ and that $M$ is minimal normal in $G$. Again, $M$ is abelian. In fact, if $M$ is non-solvable then $M$ is simple by Proposition 2.10(c); so $G / M \mathbf{C}_{G}(M)$ is solvable and hence $G=M \times \mathbf{C}_{G}(M)$ because $G$ is perfect; but this gives $\mathbf{O}^{\pi^{\prime}}(G)<G$, a contradiction.

If $M$ has a complement in $G$, then every character $\lambda \in \operatorname{Irr}(M)$ extends to $I_{G}(\lambda)$. If $M$ has no complement in $G$, then $M \leq \Phi(G)$ and so $B \leq \mathbf{F}(G)$; it follows that $M$ is a 2-group. Recall that $G / M \simeq \mathrm{SL}_{2}\left(p^{a}\right)$ contains just one involution and that a Sylow 2-subgroup $Q$ of $\mathrm{SL}_{2}\left(p^{a}\right)$ is a generalized quaternion group. An application of [8, V.25.3] yields that the Schur representation group of $Q$ is $Q$ itself; as a consequence, for each $\lambda \in \operatorname{Irr}(M)$, the group $I_{G}(\lambda) / \operatorname{ker}(\lambda)$ splits over its central subgroup $M / \operatorname{ker}(\lambda)$, and again $\lambda$ extends to $I_{G}(\lambda)$. Now, using Gallagher's theorem 
$([10,(6.17)])$ and the subgroup structure of $G / M$, one readily shows that there is a $\chi \in \operatorname{Irr}(G)$ such that two distinct primes in $\pi$ divide $\chi(1)$, the final contradiction.

We can formulate Theorem A in a slightly different way

Theorem 2.11. Let $G$ be a group, and $\pi$ an independent set of vertices of $\Delta(G)$ with $|\pi|=3$. Then there exist normal subgroups $S$ and $C=\mathbf{C}_{G}(S)$ of $G$ such that

(a) $S$ is a 2-dimensional special or projective special linear group over a finite field $\mathbb{F}$ of characteristic p;

(b) $|C \cap S| \in\{1,2\}$ and the prime divisors of $|G / S C|$ are adjacent to all primes in $\mathrm{V}(S) \backslash\{p\}$; in particular, $G / S C$ is a $\pi^{\prime}$-group;

(c) $\mathrm{V}(C) \cap \pi=\emptyset$.

Proof. The existence of $S \unlhd G$ as in (a) follows from Theorem A; also, $|\mathbb{F}| \neq 9$. So, the Schur multiplier of $S$ has order either 1 or 2. Now Clifford Theory yields (c) and, recalling Proposition 2.7, also (b).

As an immediate consequence of Theorem A, we derive the Main Theorem of [18].

Corollary B. Let $G$ be a group, and $\pi$ a set of vertices of $\Delta(G)$ with $|\pi|=4$. Then at least two vertices in $\pi$ are adjacent in $\Delta(G)$.

Proof. Assume, working by contradiction, that $\pi$ is an independent set of $\Delta(G)$ with $|\pi|=4$, and let $\pi_{0}$ be a subset of $\pi$ such that $\left|\pi_{0}\right|=3$. By Theorem A, there exists a unique non-abelian composition factor $S$ of $G$ such that $\pi(S) \cap \pi_{0} \neq \emptyset$ (and in fact, $\pi_{0} \subseteq \pi(S)$ ). Since it is possible to choose the set $\pi_{0}$ in several ways (where two distinct choices produce subsets that have a non-empty intersection), we get a clear contradiction.

Corollary C. Let $G$ be a group such that $\Delta(G)$ is connected. Then the diameter of $\Delta(G)$ is at most three.

Proof. Assume, working by contradiction, that $u$ and $v$ are two vertices of $\Delta(G)$ having distance 4 ; let $u=p_{1}-p_{2}-p_{3}-p_{4}-p_{5}=v$ a minimal path linking them. Then $\left\{p_{1}, p_{3}, p_{5}\right\}$ is independent in $\Delta(G)$ and we can apply Theorem 2.11: using its notation, we easily deduce that $C$ must be abelian (every vertex of $\Delta(C)$ is adjacent to all vertices of $\Delta(S)$, as one readily checks in the direct product $S / Z \times C / Z$, where $Z=C \cap S)$. Let $\pi_{+}=\pi\left(p^{a}+1\right)$ and $\pi_{-}=\pi\left(p^{a}-1\right)$ and (say) $p \in\left\{p_{1}, p_{3}\right\} \subseteq \pi_{+} \cup\{p\}, p_{5} \in \pi_{-}$. Hence, $d_{\Delta(G)}\left(p_{2}, p_{5}\right)>2$ and $p_{2} \neq p \neq p_{5}$. If $S C<G$, then we get a contradiction by Proposition 2.7. If $G=S C$, then $\Delta(G)=\Delta(S)$, again a contradiction by Proposition 2.6.

Corollary D. Let $G$ be a group. Then $\Delta(G)$ has three connected components if and only if $G \simeq \operatorname{PSL}_{2}\left(2^{a}\right) \times A$ with $A$ abelian.

Proof. Take a vertex $p_{i}, i=1,2,3$, in each connected component of $\Delta(G)$; then $\pi=\left\{p_{1}, p_{2}, p_{3}\right\}$ is independent in $\Delta(G)$. Using Theorem 2.11 and its notation, we easily deduce that $C$ is abelian and, by Proposition 2.7, that $G=S C$. The characteristic of $S$ must be 2, otherwise two vertices in $\pi$ belong to the same connected component of $\Delta(S)$ and then of $\Delta(G)$. We conclude that $G=S \times C$. 


\section{REFERENCES}

[1] Z. Akhlaghi, C. Casolo, S. Dolfi, K. Khedri, E. Pacifici, On the character degree graph of solvable groups, Proc. Amer. Math. Soc., http://dx.doi.org/10.1090/proc/13879, to appear.

[2] C. Casolo, Some linear actions of finite groups with $q^{\prime}$-orbits, J. Group Theory 13 (2010), 503-534.

[3] C. Casolo, S. Dolfi, Product of primes in conjugacy class sizes and irreducible character degrees, Israel J. Math. 174 (2009), 403-418.

[4] C. Casolo, S. Dolfi, E. Pacifici, L. Sanus, Groups whose character degree graph has diameter three, Israel J. Math. 215 (2016), 523-558.

[5] S. Dolfi, Large orbits in coprime actions of solvable groups, Trans. Amer. Math. Soc. 360 (2008), 135-152.

[6] S. Dolfi, A. Moretó, G. Navarro, The groups with exactly one class of size a multiple of $p$, J. Group Theory 12 (2009), 219-234.

[7] L. Dornhoff, Group Representation Theory, M. Dekker, New York, 1971.

[8] B. Huppert, Endliche Gruppen I, Springer, Berlin, 1983.

[9] B. Huppert, Character theory of finite groups, De Gruyter, Berlin, 1998.

[10] I.M. Isaacs, Character Theory of Finite Groups, AMS Chelsea Publishing , 1976.

[11] I.M. Isaacs, Finite Group Theory, Graduate Studies in Mathematics 92, A.M.S., Providence, 2008.

[12] C. Köhler, H. Pahlings, Regular orbits and the $k(G V)$-problem, in: Groups and Computation III: Proceedings of the International Conference at the Ohio State University, June 15-19, (1999, 2001), 209-228.

[13] M.L. Lewis, D.L. White, Connectedness of degree graphs of nonsolvable groups, J. Algebra 266 (2003), 51-76.

[14] M.L. Lewis, D.L. White, Diameters of degree graphs of nonsolvable groups, J. Algebra 283 (2005), 80-92.

[15] M.L. Lewis, D.L. White, Diameters of degree graphs of nonsolvable groups II, J. Algebra 312 (2007), 634-649.

[16] M.L. Lewis, J.K. McVey, Character degree graphs of automorphism groups of characteristically simple groups, J. Group Theory, 12 (2009), 387-391.

[17] O. Manz, T.R. Wolf, Representations of solvable groups, Cambridge University Press, Cambridge, 1993.

[18] A. Moreto, P.H. Tiep, Prime divisors of character degrees, J. Group Theory 11 (2008), 341356.

[19] P.P. Pálfy, On the character degree graph of solvable groups. I. Three primes, Period. Math. Hungar 36 (1998), 61-65.

[20] D.L. White, Character degrees of extensions of $\mathrm{PSL}_{2}(q)$ and $\mathrm{SL}_{2}(q)$, J. Group Theory 16 (2013), 1-33.

[21] M. Suzuki, Group Theory I, Springer Verlag, New York, 1982.

[22] J. Zhang, A note on character degrees of finite solvable groups, Comm. Algebra 9 (2000), $4249-4258$.

Silvio Dolfi, Dipartimento di Matematica e Informatica U. Dini, Università degli Studi di Firenze, viale Morgagni 67/A, 50134 Firenze, Italy.

E-mail address: dolfi@math.unifi.it

Khatoon Khedri, Department of Mathematical Sciences, ISFAHAN University OF TeChNOLOGy, 84156-83111 Isfahan, Iran.

E-mail address: k.khedri@math.iut.ac.ir

Emanuele Pacifici, Dipartimento di Matematica F. Enriques, Università degli Studi di Milano, via Saldini 50, 20133 Milano, Italy.

E-mail address: emanuele.pacifici@unimi.it 